\title{
BUDDE, Gunilla, CONRAD, Sebastian, JANZ, Oliver, Transnationale Geschichte. Themen, Tendenzen und Theorien
}

\section{Michel Espagne}

\section{(2) OpenEdition \\ Journals}

Édition électronique

URL : http://journals.openedition.org/ifha/499

DOI : $10.4000 /$ ifha.499

ISSN : 2198-8943

Éditeur

IFRA - Institut franco-allemand (sciences historiques et sociales)

Référence électronique

Michel Espagne, «BUDDE, Gunilla, CONRAD, Sebastian, JANZ, Oliver, Transnationale Geschichte. Themen, Tendenzen und Theorien », Revue de I'IFHA [En ligne], Date de recension, mis en ligne le 01 janvier 2007, consulté le 22 septembre 2020. URL : http://journals.openedition.org/ifha/499; DOI : https://doi.org/10.4000/ifha.499

Ce document a été généré automatiquement le 22 septembre 2020.

(CIFHA 


\title{
BUDDE, Gunilla, CONRAD, Sebastian, JANZ, Oliver, Transnationale Geschichte. Themen, Tendenzen und Theorien
}

\author{
Michel Espagne
}

La vingtaine de contributions rassemblées par G.B., S.B. et O.J. à l'occasion du 65ème anniversaire de Jürgen Kocka mettent en évidence la nécessité de pratiquer de nos jours une histoire transnationale et abordent les problèmes de cette universalisation de la perspective. Dans un essai optimiste N. ZEMON DAVIS observe le progrès d'une globalisation qui nous permet de lire désormais Ibn Khaldun, inconnu en dehors du monde arabe jusqu'au XIXe s. Les États-nations ne sont pas morts mais dépassés par l'établissement de macro-régions qui annoncent une globalisation (Michael MANN). Peut-être même est-il possible de considérer que l'ère d'une histoire territorialisée arrive progressivement à son terme (Ch. S. MAIER). Il est vrai que la mécanique de constitution et de désagrégation des empires montre qu'une phase de perception transnationale de l'histoire se situe précisément dans le cadre des ensembles impériaux (J. OSTERHAMMEL). Analyser l'écart économique qui s'est creusé entre la Chine et l'Europe du XIVe au XIXe s. implique une perspective universaliste (P. K. O'BRIEN), même si les historiographies globalisantes restent enracinées dans des traditions nationales, comme le prouve l'évolution de l'historiographie allemande lors de sa professionnalisation au XIXe s. (G. G. IGGERS). En plaidant pour les « subaltern studies ", on opte tout naturellement pour une historiographie décalée par rapport à l'ethnocentrisme européen ou nord-américain (P. CHATTERJEE). Il est vrai que les historiens européens, qui abordent la question des histoires nationales différemment selon qu'ils viennent de l'Est ou de l'Ouest du continent lui-même, manifestent un certain ostracisme à l'égard de certains espaces qui le composent, comme la Méditerranée par exemple (H. KAELBLE). Il est également vrai qu'on ne traite pas des différents secteurs de l'espace européen et par exemple de l'Europe orientale avec les mêmes outils méthodologiques et en se concentrant sur les mêmes objets que pour 
l'ouest de l'Europe (M. HILDERMEIER). Bien que cette disparité soit de nature à rendre prudent sur la question du comparatisme comme forme d'histoire transnationale par excellence, la défense du comparatisme et de ses variantes récentes, comme l'histoire croisée, reste d'actualité (H. G. HAUPT). On ne peut que partager en tout cas l'agacement devant le paradigme du "Sonderweg " (J. J. SHEEHAN) qui fige nécessairement les historiographies dans les cadres nationaux. Ceux-ci trouvent au demeurant des défenseurs insistant sur la pertinence quantitative des enquêtes qui peuvent être plus facilement conduites dans de tels cadres (H.-U. WEHLER), eux-mêmes observables seulement de manière comparative (D. LANGEWIESCHE). Même transnationale l'histoire ne doit pas s'émanciper du particulier et de ses paradoxes : alors que l'histoire juive met l'accent sur les phénomènes identitaires et la construction de la nation (S. VOLKOV), l'histoire de la Shoah tend de plus en plus à devenir l'objet d'une mémoire transnationale, qui peut aussi servir de prétexte à la mise en évidence d'expériences locales sans lien direct avec l'anéantissement des juifs d'Allemagne ou d'Europe orientale (M. ZIMMERMANN). D'autres histoires spécifiques seraient par exemple l'histoire des idées, dont la circulation universelle fait d'autant plus problème qu'elle repose trop souvent sur l'opposition entre un niveau noble et un niveau trivial (E. ROTHSCHILD). L'histoire culturelle, lorsqu'elle aspire à l'universalité, est confrontée à l'obsolescence d'analyses culturelles transnationales en termes de structures (P. JELAVICH). Quant aux histoires commerciales fondées par exemple sur l'analyse de magasins ou de compagnies, elles font appel à des dimensions multiples lorsqu'elles tentent d'aborder un niveau global (V. DE GRAZIA), même s'il est évident que l'histoire des affaires et des banques est par définition transnationale (G. D. FELDMAN). L'histoire $\mathrm{du}$ monde ouvrier peut gagner dans une globalisation de son champ une meilleure accentuation de paradigmes anthropologiques souvent ignorés lorsqu'elle s'enferme dans des cadres européens (M. VAN DER LINDEN). C'est encore par la biais de l'anthropologie que la microhistoire peut elle aussi paradoxalement se globaliser (D. W. SABEAN). Quant à l'approche historiographique désormais classique en termes de " lieux de mémoire ", elle aboutit à des élargissements des espaces étudiés mais reconstitue des ensembles identitaires (É. FRANÇOIS). Le volume s'achève enfin sur une interrogation très opportune : le rôle d'un journalisme globalisé dans la vie des sociétés démocratiques (J. KEANE).

2 Le mérite de cet ouvrage qui aborde de nombreux domaines historiographiques sous l'angle de la globalisation est d'offrir une profusion de perspectives nouvelles. C'est aussi une limite et l'on aurait pu souhaiter au-delà de la dispersion la définition d'un certain nombre d'axes forts, éventuellement révisables, susceptibles d'éclairer le passage de relais d'une histoire sociale comparée des sociétés nationales à une historiographie dont le caractère transnational reposerait moins sur une fatalité que sur un choix. Quoi qu'il en soit, il est clair qu'on apprend beaucoup de choses à explorer ce vaste spectre de positions historiographiques possibles.

Michel ESPAGNE (Centre National de la Recherche Scientifique) 